\title{
Development Trend Research and Strategies Analysis on Generative Teaching Based on Fuzzy Matrix and Cluster Analysis
}

\author{
Bofei Xie \\ Wenzhou Vocational and Technical College, Wenzhou, China \\ xiebofei1@126.com
}

Keywords: innovation; generation; P.E. teaching; associate membership; fuzzy matrix; cluster analysis

\begin{abstract}
Innovative education is the basic education mode of innovative talent cultivation, and it's also the urgent needs of vocational college application-oriented talents. According to the the concept of innovation education, the higher vocational colleges cognition to the generative P.E. teaching, it stimulates the students' creative thinking in the view of generative thinking and constructs the fuzzy matrix which focus on generative teaching target attribution, at this degree, based on the associate membership to determine the classification relationship of P.E. teaching, that will timely feedback and fuzzy cluster analyze the generative interactive state in the P.E. teaching, further through the data mining to adjust the teaching idea and teaching behavior, it continuously breaks through the old mode of physical education and constructs P.E. teaching mode of innovation mechanism, providing theory basis and the practice path for the P.E. teaching in higher vocational
\end{abstract} colleges to a certain extent.

\section{Introduction}

Innovative education is an important part of quality education, and also an innovation of the existing education, in the teaching process, it can be free from the limitations of traditional education, to find the answers from multidirectional, multiangle levels when encounter problems. In teaching, the more important thing is to cultivate innovative consciousness and spirit of students by new learning methods and thinking mode[1,2].At the same time,the generative P.E. teaching is also a constantly changing process, to guide the students constructing their own P.E.rsonal characteristics of the learning plan, to pay attention to interactive communication between teachers and students, to improve students' quality from the knowledge, skills, emotions, values, outlook on life and other kinds of asP.E.ct. In the teaching process, the generative P.E. Teaching that under the concept of innovation education focus on individual characteristics, targeted analysis and stimulate creative thinking at the same time[3]. First of all it should be able to clear the stimulated motivation, encourage students to develop their own creative thinking and behavior, clear the teachers' motivate formation in the P.E. teaching process, encourage students to achieve their learning goals through the combination of interior and exterior[4,5]. When the teacher who introduce the P.E. knowledge teaches the basic knowledge of relevant projects, they can be accepted totally,exactly, divergently and correctly understood by the students in the process, and the students can use the innovative thinking mode to consider the problems in the teaching and create active learning measures.

\section{The Cognition of Generative P.E. Teaching Under the Concept of Innovative Education}

A. The connotation of Innovative educational concept

Innovative education encourages students to create, question and critique in the learning process, that inquiries students' indeP.E.ndent thinking ability and excavates their maximum potential[6]. At the same time, it integrates students' enthusiasm of learning and practice ability under the new teaching methods. The basic value orientation of innovation educational concept is mainly to cultivate the students' innovation spirit and innovation ability, then to excavate the potential of students and emphasize students' active learning initiative, as shown in the following figure. 


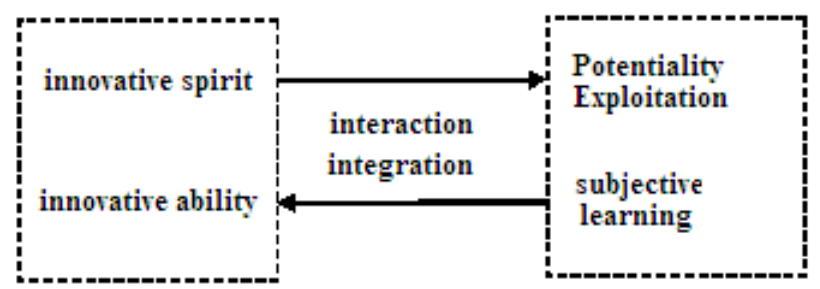

Figure 1. The connotation of Innovative educational concept

Figure 1 indicates that innovative educational concept focuses on the spirit and ability of innovation, while emphasizes the subjective learning, it is one of the core proposition of modern education that can make the entire teaching emerge new understanding and help students grasp learning points in the process, overcome the thinking difficulties, grasp the knowledge and conscious thought both in macro and micro, and better integrate the learning content .

B. The cognition of Generative P.E. Teaching

Generative P.E. Teaching has received particular attention from Higher Vocational colleges which focus on the cultivation of career diathesis, it's mainly because that generative P.E. teaching focus on the learning process, it emphasizes the students' motivation and willingness, pays attention to the improvement of student synthetic quality in the the entire sports teaching process. In the actual P.E. Teaching, ordinary physical teachers and students have almost the same the cognition on the generative P.E. Teaching, the concrete results are shown below.

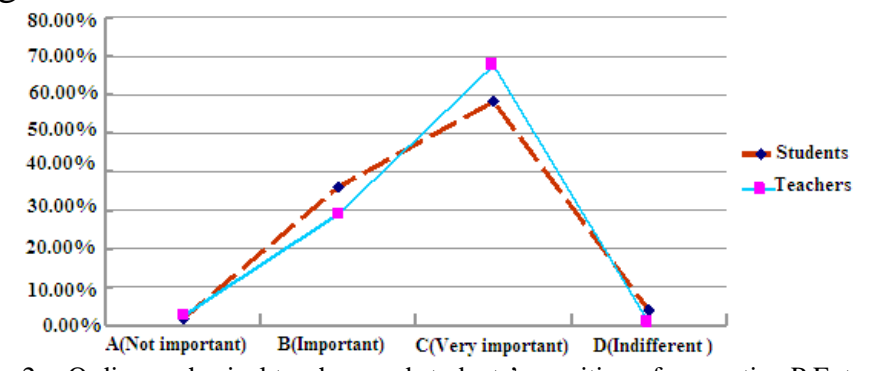

Figure 2. Ordinary physical teachers and students' cognition of generative P.E. teaching

As shown in Figure 2 , whether the teachers or students , their cognitions on the generative P.E. teaching are tending close, more than half of the teachers and students that generative P.E. Teaching is very important; vast majority of them show enthusiasm obviously in the generative P.E. teaching process and also think of the importance of generative sports teaching; only less than one P.E.rcent of the teachers think it does not matter, and there are also only $4 \%$ of students think it so.

\section{Analysis on the target attribution mode of generative P.E. teaching}

Generative teaching is a new-tyP.E. teaching concept which formed based on dynamic classroom, the main content is that in the teacher-student interaction of reflecting the students' subjectivity, teachers can effectively set the scene to excavate students ' learning potential, emanate students' thinking, organic adjust teaching default to achieve positive development of students' P.E.rsonality, then on the basis of this to complete teaching objectives and achieve the desired teaching effect[7] .

In the actual teaching process, it's need to clear the uncertain factors of generative P.E. teaching, then teachers can focus on the students' P.E.rsonality characteristics, pay attention to the cultivation of students' comprehensive abilities on the foundation of basic abilities, clear the target attribute of generative P.E. teaching. Through the attribute analysis of construct fuzzy matrix to project teaching plan, the membership degree of generative sports teaching target attribution can be set as $\lambda$, when the horizonal $\lambda_{1}$ and $\lambda_{2}$ meet $0 \leq \lambda_{1} \leq \lambda_{2} \leq 1$. According to the job characteristics to set the teaching content of P.E.,then the single attribution which divided by the $\lambda_{2}$ must be the subclass of P.E. Teaching job which divided by the $\lambda_{1}$, for example, in the track and field physical teaching,set $x_{1}, X_{2},{ }^{x_{3}}$ as three samples,and their attribute impact index are $1 、 0.6 、 0.4$,then the expression of fuzzy matrix when $X=\left\{x_{1}, x_{2}, x_{3}\right\}$ is as follows: 


$$
R=\left[\begin{array}{ccc}
1 & 0.4 & 0.6 \\
0.4 & 1 & 0.4 \\
0.6 & 0.4 & 1
\end{array}\right]
$$

As shown in the formula (1), the different attribute indexs are used to build fuzzy matrix, which mainly involves interaction and effect of students ' physical quality in the track and field sports teaching, first of all, teachers focus on the students ' basic knowledge, basic skills, learning motivation and so on, then evaluate the uncertain factors that effect teaching, as shown in the following figure.

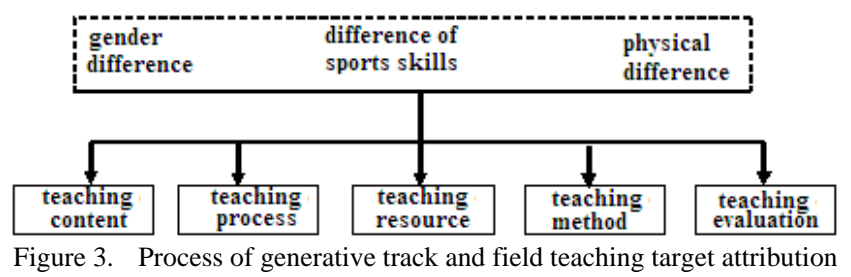

As shown in Figure 3 , in track and field teaching, it focus on the hierarchy of teaching content and integrity of the teaching process, basic skills and knowledge are as the premise of generative teaching, through orderly or disorderly generative interaction and evaluation,then it can determine the uncertain factors in the actual teaching process, take advantage of the classroom teaching opportunity to eliminate uncertain negative factors, that in formula(1), $\mathrm{X}$ is classified from $\mathrm{R}$, when $0.6\langle\lambda \leq 1$,then the results are shown as follows:

$$
R_{\lambda}=\left[\begin{array}{lll}
1 & 0 & 0 \\
0 & 1 & 0 \\
0 & 0 & 1
\end{array}\right]
$$

If we combines formula (2) and attribute degree, then the excitation can be defined as a process of mobilizing students' learning positivity, that is a reP.E.at learning process that through obtaining the object of the the development of generative track and field teaching of innovative thinking and vigorous promotion of academic P.E.rformance, guiding and changing the students' learning behavior. it is found $X=\left\{x_{1}\right\} \cup\left\{x_{2}\right\} \cup\left\{x_{3}\right\}$, namely $x_{1}, x_{2}, x_{3}$ are different tyP.E.s, when $0.4\langle\lambda \leq 0.6$, the results shown in the following formula :

$$
R_{\lambda}=\left[\begin{array}{lll}
1 & 0 & 1 \\
0 & 1 & 0 \\
1 & 0 & 1
\end{array}\right]
$$

By formula (3) shows $X=\left\{x_{1}, x_{3}\right\} \cup\left\{x_{2}\right\}$, namely $x_{1}, x_{3}$ into one class, $x_{2}$ into another one, to make the actual teaching create one kind of power that can continue to maintain or be about to change students way of thinking and learning quality, which can make every student in the class could be able to give full play their learning potential, by affecting the learning needs and learning motivation of students to achieve the purpose of effecting each student's learning behavior, to strengthen the students in the track and field courses in the learning process.when $0 \leq \lambda \leq 0.4$, after transformation it can be obtained:

$$
R_{\lambda}=\left[\begin{array}{lll}
1 & 1 & 1 \\
1 & 1 & 1 \\
1 & 1 & 1
\end{array}\right]
$$

By formula (4) shows $X=\left\{x_{1}, x_{2}, x_{3}\right\}$, namely, $x_{1}, x_{2}, x_{3}$ are in a same class. That is to say, students' learning desire dominate the behavior of students in the track and field course learning, then the learning motivation is produced by the need of learning, what need is that each student's subjective learning exP.E.rience and it's also an inevitable reflection for track and field courses learning. Students in the learning process formed learning goals and desire that make up an important part of composition in every student's learning, it has also became a source of innovative 
thinking and enthusiasm.so select a different $\lambda$ value can have different physical teaching job classification on the job characteristics samples.

\section{The feedback of generative interaction state and development tendency in the P.E. education}

Evidence of feedback of generative interaction state in P.E.education

In order to better timely feedback and fuzzy clustering analysis of the generative interaction in the physical teaching process,it needs data statistics which with physical class index to finish the evidence of feedback of generative interaction for the whole teaching[8]. As in track and field teaching, according to the target attribution mode and sample of generative P.E. education, that to make point prediction and interval prediction for the average and individual score of class teaching achievement, and one of classes sP.E.cific data statistics as shown in the following Table.

\begin{tabular}{|c|c|c|c|c|c|c|c|c|c|c|c|}
\hline Teacher evaluation points & 10 & 9 & 8 & 7 & 7 & 7 & 6.5 & 6 & 6 & 5.5 & 5 \\
\hline Self points & 55 & 70 & 90 & 68 & 69 & 85 & 76 & 69 & 89 & 79 & 69 \\
\hline
\end{tabular}

As shown in Table 1, in the track and field teaching, firstly it has made a planning summary for the teaching content, through the teaching evaluation to send information to the students, which can apply multimedia teaching. Set teacher evaluation points as $Y_{i}(i=1,2, \cdots, 12)$,about the mode of feedback of generative interaction state of self points $X_{i}(i=1,2, \cdots, 12)$,that accordance with the self points of teacher evaluation interaction and students track and field teaching exam scores for self coupling, the formula is :

$$
Y_{i}=\beta_{1}+\beta_{2} X_{i}+u_{i} \quad(i=1,2, \cdots, 12)
$$

Based on Table2,the data of track and field learning index statistic,the regression function of the feedback of generative interaction state is obtained:

$$
\hat{Y}_{i}=210.44-15.778 X_{i}
$$

Base on formula(6) sample regression function to calculate the sample regression value(predictive value) of track and field teaching and corresponding residual, list a box and calculate the result,as shown in Table 2.

\begin{tabular}{|l|l|l|l|l|}
\hline \multicolumn{1}{|c|}{$X_{i}$} & \multicolumn{1}{|c|}{$X_{i}$} & \multicolumn{1}{c|}{$Y_{i}$} & \multicolumn{1}{c|}{$\hat{Y}_{i}$} & \multicolumn{1}{c|}{$\hat{u}_{i}$} \\
\hline 10 & 3 & 55 & 52.66 & 2.34 \\
\hline 9 & 2 & 70 & 68.438 & 1.562 \\
\hline 8 & 1 & 90 & 84.216 & 5.784 \\
\hline 7 & 0 & 68 & 99.994 & 0.006 \\
\hline 7 & 0 & 69 & 99.994 & -9.994 \\
\hline 7 & 0 & 85 & 99.994 & 5.006 \\
\hline 6.5 & -0.5 & 76 & 107.883 & 2.117 \\
\hline 6 & -1 & 69 & 115.772 & 9.228 \\
\hline 5.5 & -1 & 89 & 115.772 & -0.772 \\
\hline 5 & -1.5 & 79 & 123.661 & 6.339 \\
\hline $\bar{x}=\frac{\sum x_{i}}{n}=\frac{84}{12}=7$ & -2 & 69 & 131.55 & 2.34 \\
\hline
\end{tabular}

As shown in the last Table,the arithmetic expression of feedback of generative interaction state is as follows.

$$
\begin{gathered}
\hat{S}_{\hat{Y}_{0}}=\sqrt{\hat{\sigma}^{2}\left[\frac{1}{n}+\frac{\left(\bar{X}_{2}-X_{20}\right)^{2}}{\sum x_{2 i}{ }^{2}}\right]}=\sqrt{69.9\left[\frac{1}{12}+\frac{(7-7.5)^{2}}{225}\right]}=2.57 \\
\hat{S}_{\hat{Y}_{0}}=\sqrt{\hat{\sigma}^{2}\left[1+\frac{1}{n}+\frac{\left(\bar{X}_{2}-X_{20}\right)^{2}}{\sum X_{2 i}^{2}}\right]}=\sqrt{699\left[1+\frac{1}{12}+\frac{(7-7.5)^{2}}{225}\right]}=8.7
\end{gathered}
$$

As the formula (7) and (8) shown, after generative feedback matching, the final result is 69.9, the whole teaching effect is obvious. Teachers do not only teach the theoretical knowledge of physical education, but also can put forward many kinds of questions, and according to the interaction of the teacher and students to give an answer for the generative questions, at the same time through the 
evaluation system to evaluate and diagnose the students' learning effect, and give these evaluation information back to the students.

The development tendency of generative P.E.teaching under the innovative education

To change sports teaching method and achieve the breakthrough of teaching achievements are qualities which the vocational College physical teachers in new time. To ultimately achieve the innovation of P.E. Teaching thought and maximize self-realization of students' learning needs are the goal of each physical teacher. Compared with the traditional education, the generative

The generative P.E. teaching has great differences at the teaching object,teaching content, teaching requirements, teaching subject, teaching methods, teaching thought, teaching method and other asP.E.cts which resP.E.ctively under the traditional teaching and the generative P.E. Teaching that under the concept of innovative education. The generative P.E. teaching which under the concept of innovative education is more of profound, flexibility, oP.E.nness and other characteristics, it can promote students' positive start point of learning and up internal driving force, enhance students' learning motivation of problem finding and positive exploration. In the physical teaching class, the students accept feedback problems and further strengthen the feedback problems that the teachers can make a further scientific judgement, students in physical education class, in order to make a scientific judgment to the old into further decision-making, new courses are intersP.E.rsed in the teaching information, students and teachers gradually cycled and formed generative target teaching control that make optimization of the physical education final effect, and have a broad development space.

\section{Conclusion}

The generative P.E. Teaching under the concept of innovative education should inspire students' creative thinking, make every student have a positive learning emotion, according to the needs of teaching to choose learning content, the students will choose to accept the learning content under self guidance, and improve the quality of teaching, promote the students' knowledge level. In the learning process of physical teaching, responsive problems were proposed and will be reflected to the teacher, through the students questioning and teachers make judgments. In short, the sports teaching should be able to keep pace with the times, emancipate the mind, always grasp the new ideas of teaching and teaching methods, stimulate students' learning thinking, which makes the sports quality education obtain greater achievements.

\section{References}

[1] XiaoHongxiang.Theoretical Analysis of the Learning style of physical education curriculum for the students against the backdrop of new curriculum standard.Journal of Xi'an Physical Education University,2011,28(6):74-78.

[2] WuBenlian.An exP.E.rimental study on the impact of self-regulated learning in acquisition efficiency in collegiate physical culture. East china normal university,2010:34-37.

[3] Cong Lin. A Probe into Applying "Study and Discussion" Learning Method in P. E. Curriculums of Colleges \& Universities. Journal of Harbin institute of physical education,2013(1):103-105.

[4] $\mathrm{Xu}$ Yan,Sun Guang. Reflection on the Methodology of P.E. Teaching. Journal of sports and science,2012,23(4):154-157.

[5] Button H. W., \& Provenzo E. F.. History of Education and Culture Americal. Inc, Englewood Cliffs, New Jeysey,2011:233-239.

[6] Swinnen, S. P.,Schmidt, R. A.,Nicholson, D. E. \& Shapiro, D. C. Information feedback for skill acquisition: Instantaneous knowledge of results degrades learning. Journal of ExP.E.rimental Psychology: Learning, Memory, and Cognition,2010(9):31-43.

[7] Greene,J. A. , \& Azevedo, R. Adolescents' use of self-regulatory processes and their relation to qualitative mental model shifts while using P.E.rmedia. Journal of Educational Computing Research, 2012(12):1-12.

[8] Chatzisarantis,N. L. D.,\& Biddle, S.J.H. Functional significance of psychological Variable that are included in the theory of planned behaviour: A self-determination theory approach to the study of attitudes, Subjective norms,P.E.rceptions of control and intentions. Journal of Social Psychology, 2009(12):78-91. 